\title{
Synthesis and luminescence of CdS quantum dots capped with a silica precursor
}

\author{
Sander F. Wuister*, Andries Meijerink \\ Department of Chemistry, Chemistry and Physics of Condensed Matter, Debye Institute, Utrecht University, Princetonplein 5 , \\ 3584 CC Utrecht, The Netherlands
}

Received 2 January 2003; received in revised form 24 April 2003; accepted 24 April 2003

\begin{abstract}
A novel synthesis method for efficiently luminescing CdS nanocrystals is reported using (3-mercaptopropyl)trimethoxysilane (MPS) as a capping agent in THF or $\mathrm{MeOH}$. The synthesis yields particles capped with a precursor for coating the particle with a silica shell and offers control over a wide range of particle sizes $(2-6 \mathrm{~nm})$ by varying the MPS concentration. The temperature dependence of the luminescence and the luminescence life time was studied and explained in terms of a donor-acceptor recombination process in which both electron and hole are trapped at low temperature. In addition to varying the emission color by controlling the particle size, nanocrystals were doped with $\mathrm{Zn}$ or $\mathrm{Cu}$ resulting in a blue shift or a red shift of the emission.
\end{abstract}

(C) 2003 Elsevier B.V. All rights reserved.

Keywords: CdS; Nanocrystals; $\mathrm{SiO}_{2}$

\section{Introduction}

Efficiently luminescing nanocrystalline semiconductors (also known as quantum dots) form an important class of luminescent materials [1]. Studies on the influence of quantum confinement on the electronic and optical properties of nanocrystalline semiconductors are of great fundamental interest [2]. Potential applications of quantum dots for light generation and as luminescent labels in biological systems have also

\footnotetext{
*Corresponding author. Physics and Chemistry of Condensed Matter, Debye Institute, Utrecht University, P.O. Box 80000 , Utrecht, TA 3508, The Netherlands. Tel.: +31-302532207; fax: + 31-30-2532403.

E-mail address: s.f.wuister@phys.uu.nl (S.F. Wuister).
}

attracted considerable attention [3,4], especially since the development of synthesis methods for quantum dots with very high quantum yields. For example, with a nonaquaeous TOP/TOPO (trioctyl phosphine/trioctyl phosphine oxide) synthesis method CdSe [5] and CdTe [6] nanocrystals quantum dots with quantum efficiencies above $50 \%$ at room temperature can be produced. In various studies procedures have been described to couple CdSe and CdTe quantum dots to biological molecules to serve as luminescent labels in biological systems $[3,4,7-10]$. In order to make the quantum dots synthesized with the TOP/ TOPO synthesis method biocompatible, the particles have to be made water soluble. Moreover, to reduce the release of toxic heavy atoms from the quantum dots the particles may be capped by a 
nontoxic capping layer. Encapsulation in a thin silica shell is a method to obtain robust water soluble quantum dots [7,11].

In this paper the synthesis and luminescence properties of $\mathrm{CdS}$ quantum dots is described. Via a relatively simple synthesis method CdS nanoparticles are synthesized in polar media (tetrahydrofuran or methanol) using (3-mercaptopropyl)-trimethoxysilane (MPS) as a capping molecule. In addition to providing a passivating layer around the CdS quantum dot, the silane groups of MPS can serve as a precursor for growth of a silica shell around the particle. Efficiently luminescing $\mathrm{CdS}$ particles are produced with quantum yields up to $20 \%$ at room temperature. The size of the nanocrystals determines the emission color. Size control is achieved by varying the MPS concentration. Alternatively, it is demonstrated how the emission color can also be varied by incorporating $\mathrm{Zn}$ (for blue shifted emission) or $\mathrm{Cu}$ (for red shifted emission) in the CdS nanocrystals.

\section{Experimental}

\subsection{Chemicals}

Tetrahydrofuran (Anhydrous 99.9\%, Aldrich), Methanol (Anhydrous, Merck), cadmiumperchlorate hydrate (Aldrich), MPS (97\%, Aldrich) hydrogen sulfide (99.5\%, Aldrich), copper chloride dihydrate $(99 \%$ Aldrich), zinc acetate $(98+\%$, Aldrich).

\subsection{Synthesis}

In a dry nitrogen atmosphere a variable amount of MPS is added to $20 \mathrm{ml} 10^{-3} \mathrm{M} \mathrm{Cd}\left(\mathrm{ClO}_{4}\right)_{2}$ in either tetrahydrofuran (THF) or methanol $(\mathrm{MeOH})$. Under stirring $0.5 \mathrm{ml}(2.3 \times$ $10^{-5} \mathrm{~mol}$ ) $\mathrm{H}_{2} \mathrm{~S}$ (corresponding to an excess of $15 \%$ ) is added through a septum with a syringe at room temperature. The final color of the solution after addition of $\mathrm{H}_{2} \mathrm{~S}$ varies from deep yellow for $40 \mu \mathrm{MPS}$ (giving a $0.01 \mathrm{M}$ MPS solution) to colorless for an MPS concentration of $0.4 \mathrm{M}(1600 \mu \mathrm{l})$. Particle sizes between 2 and
$6 \mathrm{~nm}$ can be obtained this way. Unless mentioned otherwise copper and zinc salts were added to the $\mathrm{Cd}\left(\mathrm{ClO}_{4}\right)_{2}$ solution before addition of $\mathrm{H}_{2} \mathrm{~S}$. To prevent the particles from premature flocculation, dry solvents were used. In the presence of too much water, uncontrolled polymerization of the alkoxysilane groups occurs, leading to flocculation of the quantum dots. In dry solvents the particles were stable for months.

\subsection{Apparatus}

Emission and excitation spectra were recorded on a SPEX Fluorolog spectrofluorometer, equipped with two monochromators (doublegrating, $0.22 \mathrm{~m}$, SPEX 1680, model F2002) and a $450 \mathrm{~W}$ Xenon lamp as the excitation source. Absorption spectra were measured using a Perkin-Elmer Lambda $16 \mathrm{UV} /$ vis spectrometer. Timedependent measurements were performed using the third harmonic $(355 \mathrm{~nm})$ of a quanta-ray DCR $\mathrm{Nd}^{3+}$ :YAG laser as an excitation source. The emission light was guided through a fiber optic cable to a monochromator (Acton SP-300i, $0.3 \mathrm{~m}$, 150 lines $\mathrm{mm}^{-1}$ grating, blazed at $\left.500 \mathrm{~nm}\right)$. The signals were recorded using a thermoelectrically cooled photo-multiplier tube in combination with a Tektronix 2430 oscilloscope. Temperature-dependent PL emission and lifetime measurements were recorded using a liquid helium flow-cryostat equipped with a sample heater to stabilize the temperature at different temperatures between $4 \mathrm{~K}$ and room temperature. Samples were first cooled to $4 \mathrm{~K}$ and slowly heated to room temperature.

\section{Results and discussion}

\subsection{Optical spectroscopy of $C d S$}

The concentration of the MPS capping agent has a large influence on the final size of the CdS nanocrystals. Under otherwise identical synthesis conditions CdS particles were grown in methanol or THF using MPS concentration varying between 0.01 and 0.4 M. In Fig. 1 the absorption spectra for CdS particles prepared in methanol are shown for five different MPS concentration. The spectra 


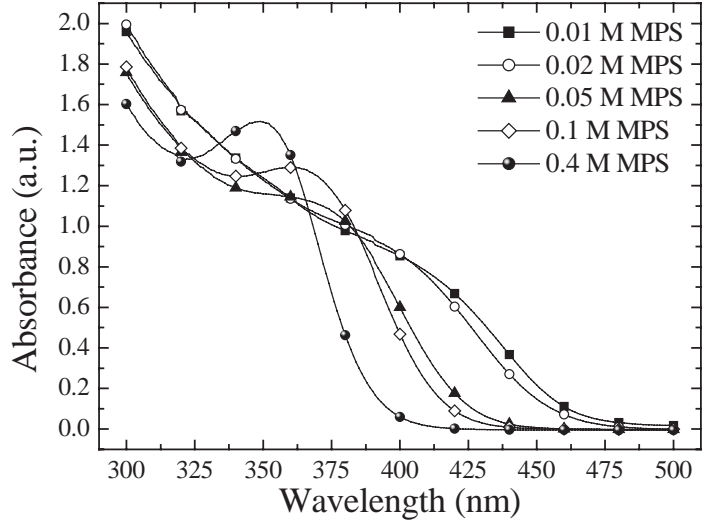

Fig. 1. Absorption spectra of CdS NCs synthesized in methanol with different concentrations of MPS (between $0.01 \mathrm{M}(40 \mu \mathrm{l})$ and $0.4 \mathrm{M}(1600 \mu \mathrm{l}))$.

show a clear shift of the absorption onset to shorter wavelengths as the MPS concentration is increased. From the position of the absorption edge the mean particle size can be determined using the well-established relation between particle size and absorption onset [12,13]. In Table 1 the results are summarized. In methanol the particle size increases from $2.2 \mathrm{~nm}$ for the highest MPS concentrations to $4.6 \mathrm{~nm}$ for the lowest MPS concentrations used. For NCs made in THF larger particles were obtained. Also the particle size distribution narrows as the concentration of the MPS in increased. To understand the size variation one should consider the growth mechanism of nanocrystals in the presence of a capping agent. It is generally accepted that the capping agent coordinates the cations. The bonding should neither be too strong nor too weak to allow for dynamic coupling in which the coordinating molecules attach and de-attach from the growing nanocrystals. In this way the particle can grow to final size which is determined by the type and the concentration of the capping agent. If the binding between the cations and the capping agent becomes stronger, the particles reach smaller final sizes. In the present situation the particles grow to larger sizes in the lower concentration regime of capping agent. As the concentration is lower, the incomplete capping of the surface by capping molecules allows for a faster particle growth.
Table 1

Quantum efficiencies and particle sizes of CdS NCs for synthesis in $\mathrm{MeOH}$ and THF for different concentrations of MPS

\begin{tabular}{lrllll}
\hline$[\mathrm{MPS}](\mathrm{mol} / \mathrm{l})$ & \multicolumn{1}{l}{$\mathrm{MeOH}$} & & \multicolumn{2}{l}{ THF } \\
\cline { 2 - 3 } \cline { 6 - 6 } & QE $(\%)$ & Size $(\mathrm{nm})$ & & QE $(\%)$ & Size $(\mathrm{nm})$ \\
\hline 0.01 & $1.9 \pm 0.1$ & $4.6 \pm 0.1$ & & $0.7 \pm 0.1$ & $6.5 \pm 0.1$ \\
0.02 & $4.7 \pm 0.2$ & $4.4 \pm 0.1$ & & $0.8 \pm 0.1$ & $6.2 \pm 0.1$ \\
0.05 & $10.5 \pm 0.5$ & $3.2 \pm 0.1$ & & $15.9 \pm 0.8$ & $4.8 \pm 0.1$ \\
0.1 & $9.9 \pm 0.5$ & $2.8 \pm 0.1$ & & $15.6 \pm 0.8$ & $4.6 \pm 0.1$ \\
0.4 & $9.5 \pm 0.5$ & $2.3 \pm 0.1$ & & $18.9 \pm 0.9$ & $4.1 \pm 0.1$ \\
\hline
\end{tabular}

Increasing the capping agent concentration results in a better shielding of the particle and hampers the growth resulting in a smaller final particle size. Previously a similar effect was demonstrated for hexanethiol as a capping agent [14].

Luminescence spectra (excitation and emission) are shown in Fig. 2 for CdS particles of different sizes. The onset of the excitation spectra is in good agreement with the absorption onsets in Fig. 1, as expected. For wavelengths below $300 \mathrm{~nm}$ the excitation spectra show a drop, while the absorption spectra still show an increase in the optical density. This indicates that the stronger absorption at shorter wavelengths does not result in efficient emission. This is often observed and may be caused by absorption of UV radiation by for example capping agent molecules. The absorption spectrum of MPS indeed shows a strongly increasing absorption below $325 \mathrm{~nm}$. Absorption of UV radiation by absorbing species other than the quantum dots is not followed by emission of the quantum dots and explains the decrease in the excitation spectrum below $300 \mathrm{~nm}$. The emission spectra are broad band emissions extending over a large part of the visible region and shift to higher energies as the particles are smaller. The shift of the emission band is smaller than the shift of the absorption onset (total shift of the emission is almost $3000 \mathrm{~cm}^{-1}$ vs. over $4000 \mathrm{~cm}^{-1}$ for the absorption spectrum of the same samples). The smaller shift is in line with the assignment to a trap-related type emission. Both the electron and hole are trapped in shallow traps and recombination of the donor acceptor pair gives rise to 


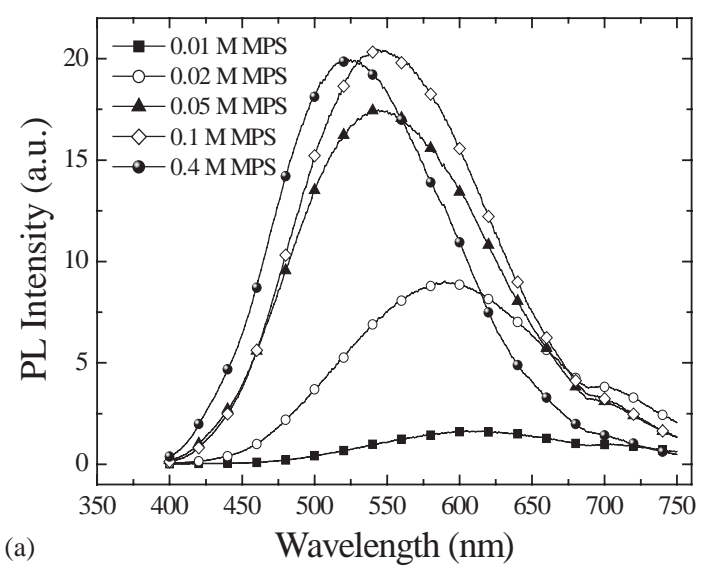

(a)

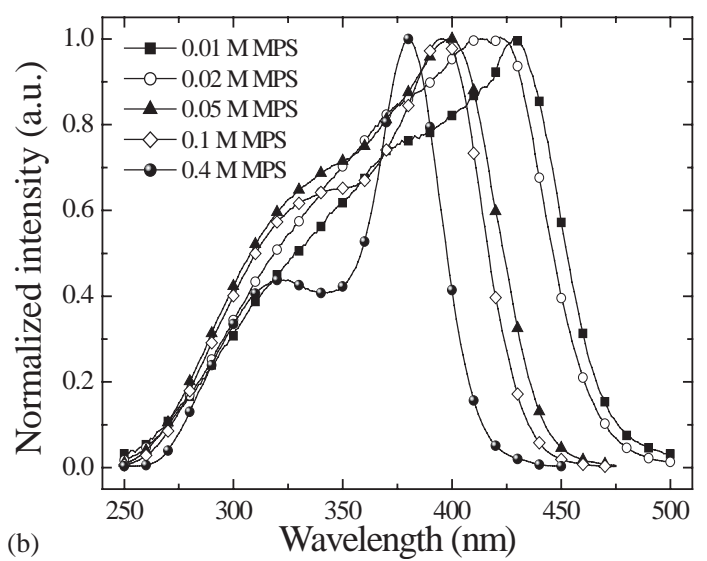

Fig. 2. (a) Room temperature emission $\operatorname{spectra}\left(\lambda_{\mathrm{ex}}=350 \mathrm{~nm}\right)$ and (b) excitation spectra (measured for the emission maximum) of CdS NCs synthesized in methanol using different concentrations of MPS (between 0.01 and $0.4 \mathrm{M}$ ).

sub-bandgap emission $[15,16]$. The shift of the acceptor and donor level usually follows the valence band and conduction band edge. As the wave function of the trapped charge carriers is less extended than for free charge carriers, the quantum size effects (and thus the energy shifts) are smaller for trapped charge carriers than for the band edges. The large spectral width of the emission band is caused by inhomogeneous broadening (due to a variation in particle size) and broadening due to electron-phonon coupling.

Quantum yields have been determined by comparison of the emission output of a solution of quantum dots with adjusted absorption and a solution of Coumarine 153 (a commercial laser dye with a $90 \%$ quantum yield) dissolved the same solvent as the $\mathrm{CdS}$ quantum dots $(\mathrm{MeOH}$ or THF). The quantum efficiencies (close to $20 \%$ for the best particles) are among the highest reported for the donor-acceptor emission in CdS quantum dots and are obtained via a relatively simple synthesis method [15]. There is a clear correlation between the quantum efficiency and the amount of MPS (see Fig. 2(a) and Table 1). Incomplete surface passivation, resulting from the use of lower MPS concentrations, gives rise to poor quantum efficiencies. Above $0.05 \mathrm{M}$ MPS the quantum efficiency is at a constant high level. The high quantum efficiencies show that the mercapto group in MPS is able to serve as a good capping to passivate the surface of $\mathrm{CdS}$ particles. In addition, the trimethoxysilane group can be used to grow a thin silica layer on the CdS surface using an alkoxysilane precursor.

Temperature-dependent emission spectra are shown in Fig. 3. As the temperature increases the emission shifts to longer wavelengths. The total shift between 4 and $300 \mathrm{~K}$ is about $300 \mathrm{meV}$. This shift is larger than the shift determined from the excitation maximum $(180 \mathrm{meV}$ to lower energies between 4 and $300 \mathrm{~K}$ ). A shift to lower energies due to narrowing of the bandgap is commonly observed for semiconductors $[17,18]$. The presently observed larger shift for the emission maximum may be related to thermal detrapping of electron or holes from the donor and acceptor sites. A redistribution over (deeper) donor-acceptor pairs at higher temperatures can explain the shift of the emission maximum. The emission intensity decreases as the temperature is raised. It is difficult to estimate the quantum efficiency at low temperatures since the liquid-to-solid transition of the solvent (176 K for methanol, $165 \mathrm{~K}$ for THF) will have a strong influence on the light path of the excitation and emission through the sample. Still, from Fig. 3(a) it is clear that there is a strong increase in the luminescence efficiency on cooling the sample to $4 \mathrm{~K}$ and the quantum efficiency may be close to $100 \%$ at $4 \mathrm{~K}$.

To follow the growth of the CdS particles as a function of time, luminescence spectra were recorded for samples taken at variable time intervals during the synthesis of CdS nanocrystals 

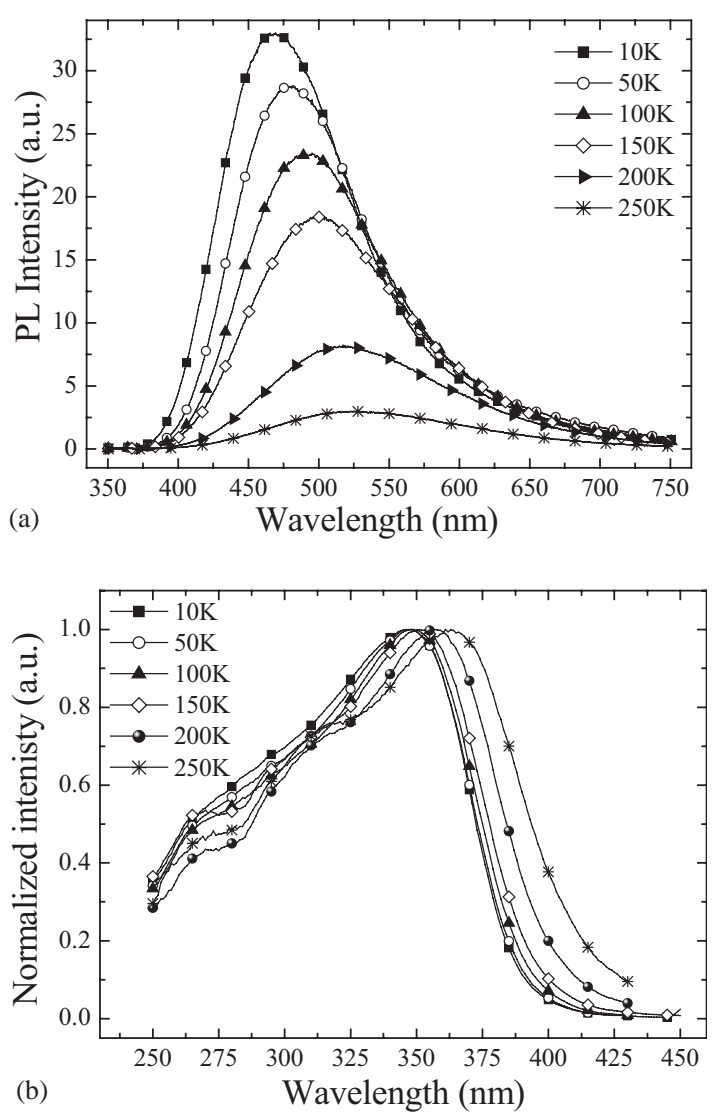

Fig. 3. (a) Temperature-dependent emission spectra $\left(\lambda_{\mathrm{ex}}=\right.$ $350 \mathrm{~nm}$ ) and excitation spectra (measured for the emission maximum) of CdS QDs synthesized in methanol with $0.4 \mathrm{M}$ MPS.

in THF. The results are shown in Fig. 4 for a synthesis with $0.05 \mathrm{M}$ MPS. After $1 \mathrm{~min}$ three sharp lines around $420 \mathrm{~nm}$ are observed on top of a broad band peaking at $525 \mathrm{~nm}$. The intensity of the broad band increases as function of time. At the same time the peak maximum shifts to longer wavelengths due to particle growth. After about $100 \mathrm{~min}$ the peak position has shifted to about $585 \mathrm{~nm}$ and the particles have reached the final size of $5 \mathrm{~nm}$. The luminescence intensity continues to increase and levels off after about $500 \mathrm{~min}$. This indicates that after the particles have reached their final size, the surface passivation by MPS still improves giving a higher luminescence quantum yield. A similar behavior is also observed for other MPS concentrations. The sharp peaks at the short
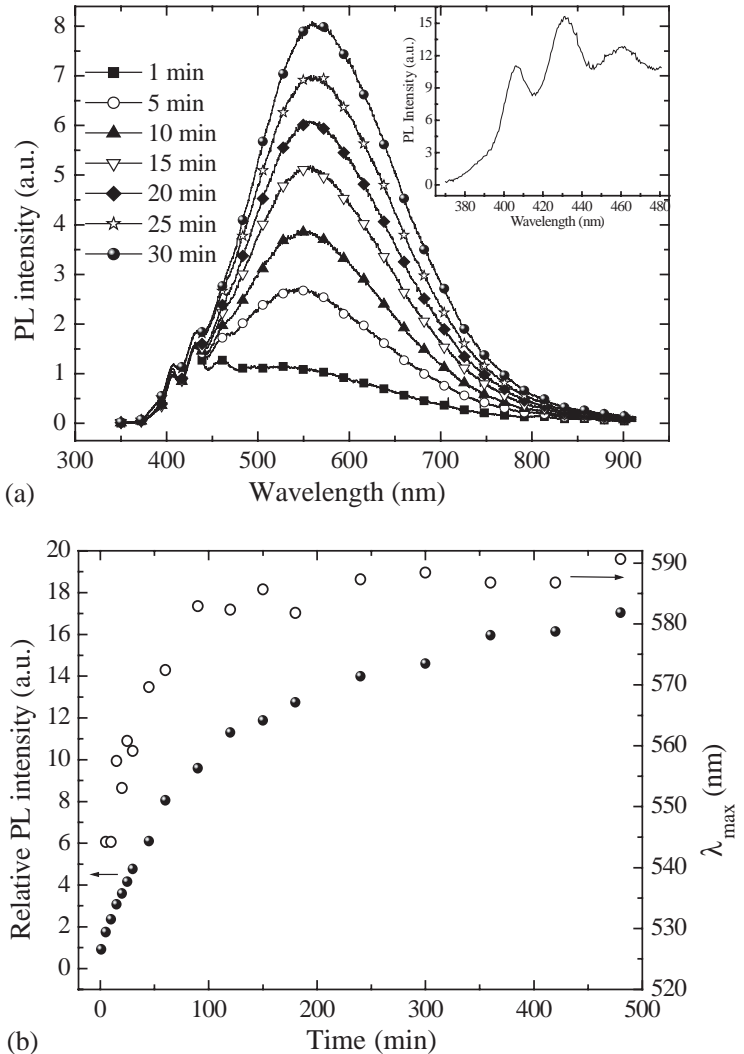

Fig. 4. (a) Emission spectra $\left(\lambda_{\mathrm{ex}}=350 \mathrm{~nm}\right)$ and (b) integrated emission intensity and peak maximum as a function of synthesis time for CdS QDs in THF ([MPS] $=0.1 \mathrm{M})$.

wavelength position of the emission band may be due to exciton emission from a small fraction of initially formed CdS particles. To verify that the sharp peaks are not due to Raman scattering, emission spectra were recorded for different excitation wavelengths. In all spectra the sharp peaks were observed at the same wavelengths (around $420 \mathrm{~nm}$ ) which shows that the peaks are not related to Raman scattering. To further investigate the nature of the line emission, lifetime measurements were performed. Indeed, the lifetime of the emission is, as expected for exciton emission, short (less than $50 \mathrm{~ns}$ ) contrary to the $\mu \mathrm{s}$ life time of the broad band emission (see also below). Luminescence spectra under higher excitation densities were performed using the third harmonic $(355 \mathrm{~nm})$ of a Nd-YAG laser. As the 


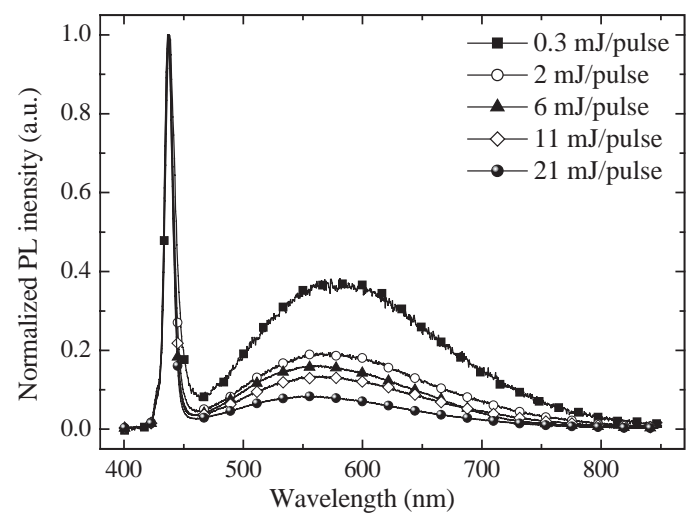

Fig. 5. Laser power dependence of emission spectra $\left(\lambda_{\mathrm{ex}}=355 \mathrm{~nm}\right)$ measured at $4 \mathrm{~K}$ for CdS NCs synthesized in THF with a MPS concentration of $0.01 \mathrm{M}$. The spectra are scaled to the peak at $440 \mathrm{~nm}$ for all laser powers.

laser power is increased the relative intensity of the line emission increases and becomes more intense than the defect emission for laser powers between 0.3 and $21 \mathrm{~mJ}$ per pulse (see Fig. 5). The increase of the relative intensity of the excitonic emission can be explained by saturation of the longer lived donoracceptor emission at high excitation densities.

The life time of the donor-acceptor emission was studied as function of temperature, particle size and emission wavelength. In Fig. 6 the results are summarized. The low temperature decay curves are almost single exponential with a life time between 5 and $20 \mu$ s, mainly depending on the amount of MPS used in the synthesis. For higher MPS concentrations better capped particles are obtained with a high quantum yield. Since the fast nonradiative decay is better suppressed for particles with a high quantum yield, a longer life time for higher MPS concentrations is expected. Above $20 \mathrm{~K}$ the decay starts to drop and at $100 \mathrm{~K}$ the life time decreased to the ns regime, to finally reach about $100 \mathrm{~ns}$ at room temperature. These results are very similar to those reported by Chestnoy et al. [19]. To explain the temperature dependence a multi-phonon relaxation model was proposed in Ref. [19]. At elevated temperatures thermal occupation of phonon modes enhances the nonradiative decay rate as described in for example Refs. [20,21]. Even though the fit to the model for multi-phonon relaxation gave a reasonably

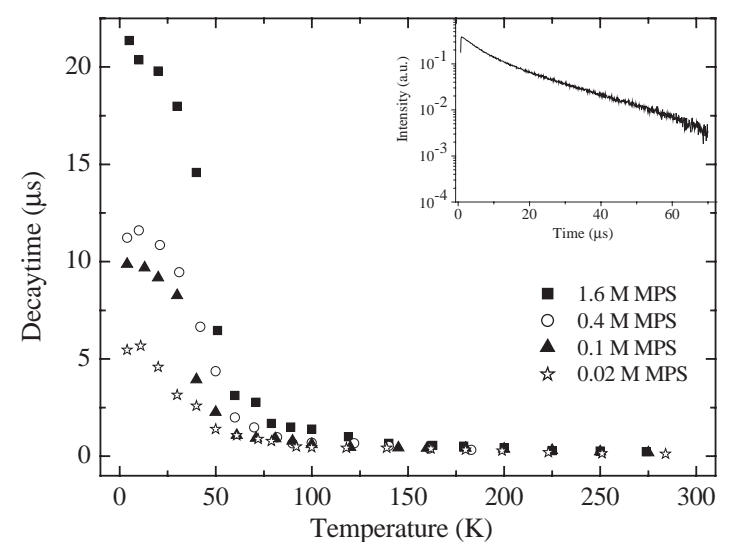

Fig. 6. Temperature dependence of the life time of the CdS emission $\left(\lambda_{\mathrm{ex}}=355 \mathrm{~nm}\right)$ for four different MPS concentrations in THF. The inset shows a typical decay curve for $4.1 \mathrm{~nm} \mathrm{CdS}$ particles at $4 \mathrm{~K}$. Lifetimes were determined for the linear tail of the logarithmic decay curve.

good agreement with the measured temperature dependence for the life time, there are several reasons why it is not likely that this type of thermal quenching is responsible for the temperature dependence of the life time. Firstly, the Huang-Rhys coupling parameter $S$ that was determined from the fits in Ref. [19] is 110. This is an unrealistically high value. Secondly, the temperature dependence of the emission intensity does not show a large drop between 20 and $100 \mathrm{~K}$ (see Fig. 3). If increased nonradiative relaxation would be responsible, the intensity has to drop at the same rate as the decay time. Rather than multiphonon relaxation we propose that the shortening of the life time is due to thermal detrapping of one of the charge carriers. As discussed above, the broad band luminescence is most likely the result of recombination of donor-acceptor pairs with shallowly trapped electrons and holes. As the temperature is raised one of the charge carriers (the one in the shallower trap) may be thermally detrapped and become more delocalized. This will increase the wave function overlap for recombination with the other trapped charge carrier and thus increase the radiative recombination rate. For this model an Arrhenius type temperature dependence of the life time is expected and from the slope of the plot of $\ln \tau$ vs. $1 / T$ the trap depth may be determined for the shallower trap. From the 


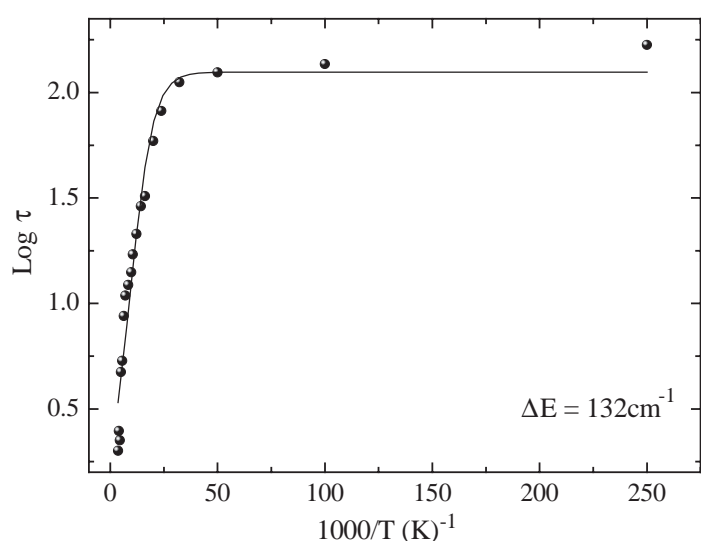

Fig. 7. Arrhenius plot of the decay time of the emission from CdS particles synthesized in a 0.1 M MPS solution in THF. The logarithm of the decay time is plotted against the $1000 / T$. The drawn line is a fit to the Arrhenius equation for an activation energy of $132 \mathrm{~cm}^{-1}$.

results in Fig. 7 a trap depth of about $132 \mathrm{~cm}^{-1}$ has been estimated.

\subsection{Luminescence of ( $\mathrm{Cd}, \mathrm{Zn}) \mathrm{S}$ and $\mathrm{CdS}: \mathrm{Cu}$}

In the previous section, the emission color was varied by tuning the band gap through the particle size. An alternative method to shift the bandgap of $\mathrm{CdS}$ is co-doping with $\mathrm{Zn}$ [22]. The bandgap increases as $\mathrm{Zn}$ is added. To shift the absorption and emission to shorter wavelengths $\mathrm{Zn}$ was incorporated in the $\mathrm{CdS}$ nanocrystals using the same synthesis method with MPS as a capping agent $(0.05 \mathrm{M})$ and using 5,10 or $15 \mathrm{~mol} \%$ of $\mathrm{Zn}$ instead of Cd. In Fig. 8 absorption and emission spectra are shown. From the absorption spectra it is clear that the bandgap increases due to the incorporation of $\mathrm{Zn}$. The narrower excitonic band observed for higher $\mathrm{Zn}$ concentrations suggests that the particle size distribution is more monodispers if $\mathrm{Zn}$ is added. The donor-acceptor emission band also shifts to shorter wavelengths as more $\mathrm{Zn}$ is incorporated. The shift of the emission maximum is $2355 \mathrm{~cm}^{-1}$ vs. $2925 \mathrm{~cm}^{-1}$ for the absorption onset. The smaller shift of the emission maximum is in line with the results presented above for the shift in the absorption and emission wavelengths by size variation. The luminescence quantum efficiency remains high
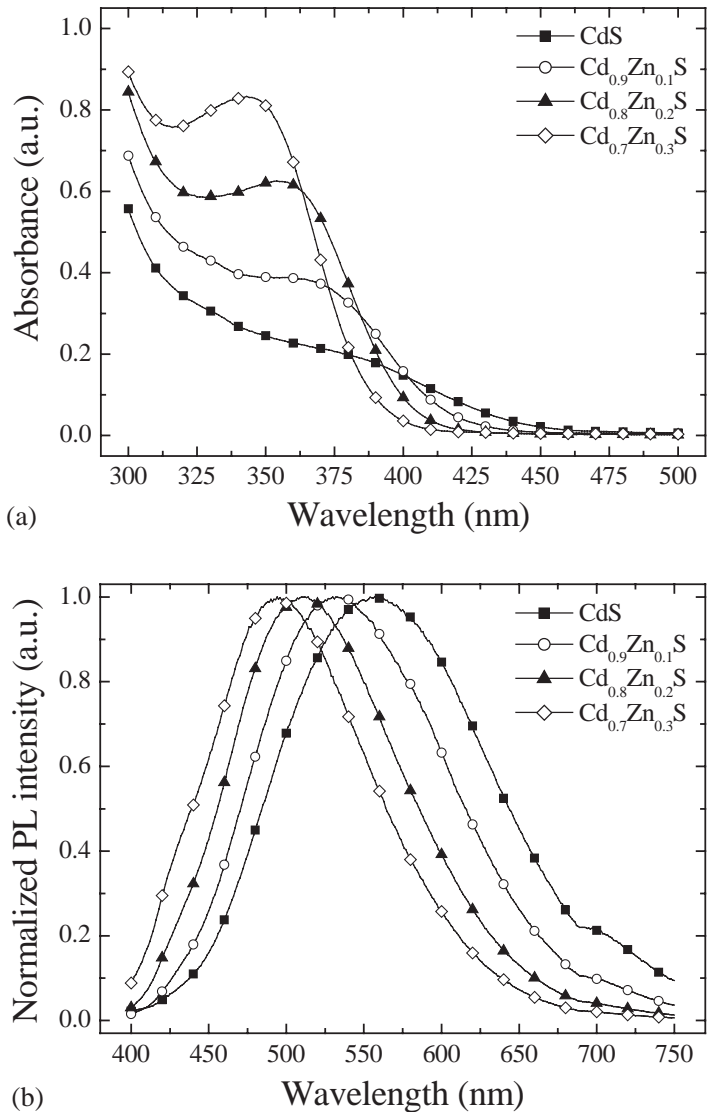

Fig. 8. (a) Absorption and (b) emission spectra $\left(\lambda_{\mathrm{ex}}=350 \mathrm{~nm}\right)$ of $\mathrm{Cd}_{(1-x)} \mathrm{Zn}_{x} \mathrm{~S}(0 \leqslant x \leqslant 0.3)$ made with a MPS concentration of $0.1 \mathrm{M}$ in THF. The Zn-precursor was added before particle formation.

(between $15 \%$ and $20 \%$ ) for the synthesis with $\mathrm{Zn}$. However, the absorption strength increases remarkably by almost a factor of four from pure $\mathrm{CdS}$ to $\mathrm{Cd}_{0.85} \mathrm{Zn}_{0.15} \mathrm{~S}$. This increase is clear from Fig. 8a where the absorption spectra are shown for the four different samples recorded for the same concentrations. At the moment this increase absorption strength is not well understood. The bandgap can also be shifted to higher energies if the $\mathrm{Zn}$ is added after the CdS particles have been formed. In Figs. 9 and 10 the absorption and emission spectra are shown for CdS nanocrystals grown in THF to which a solution of the $\mathrm{Zn}$ precursor was added 3 days after the formation of the CdS particles. The shift of the absorption and emission band for the samples with $10 \% \mathrm{Zn}$ added 

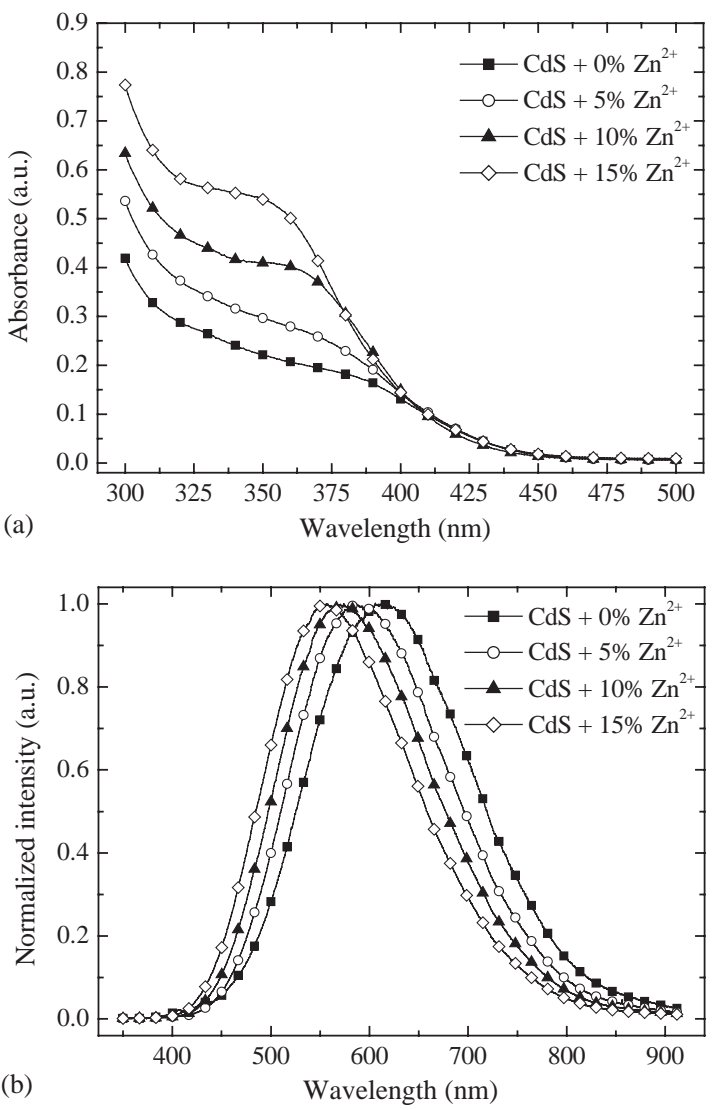

Fig. 9. (a) Absorption and (b) emission spectra $\left(\lambda_{\mathrm{ex}}=350 \mathrm{~nm}\right)$ of CdS made with a MPS concentration of $0.1 \mathrm{M}$ in THF. Three days after particle formation $0-15 \% \mathrm{Zn}$ (relative to $\mathrm{Cd}$ ) was added.

after the particle formation is slightly smaller than the shift observed in Fig. 7 (about $100 \mathrm{~cm}^{-1}$ smaller) but the fact that also by Zn-addition after the formation of CdS nanoparticles a significant shift to higher energies is observed indicates that $\mathrm{ZnS}$ does not form a capping layer on the $\mathrm{CdS}$ nanocrystals (in that case a red shift would be expected). The shift in bandgap may be explained by an exchange of $\mathrm{Cd}$ and $\mathrm{Zn}$ during the growth after the addition of the $\mathrm{Zn}$-precursor causing the formation mixed $(\mathrm{Cd}, \mathrm{Zn}) \mathrm{S}$ nanocrystals.

Incorporation of $\mathrm{Zn}$ causes a blue shift of the absorption and emission spectra. To obtain a redshifted emission, $\mathrm{Cu}$ may be added $[18,23]$. The addition of $\mathrm{Cu}$ does not strongly influence the
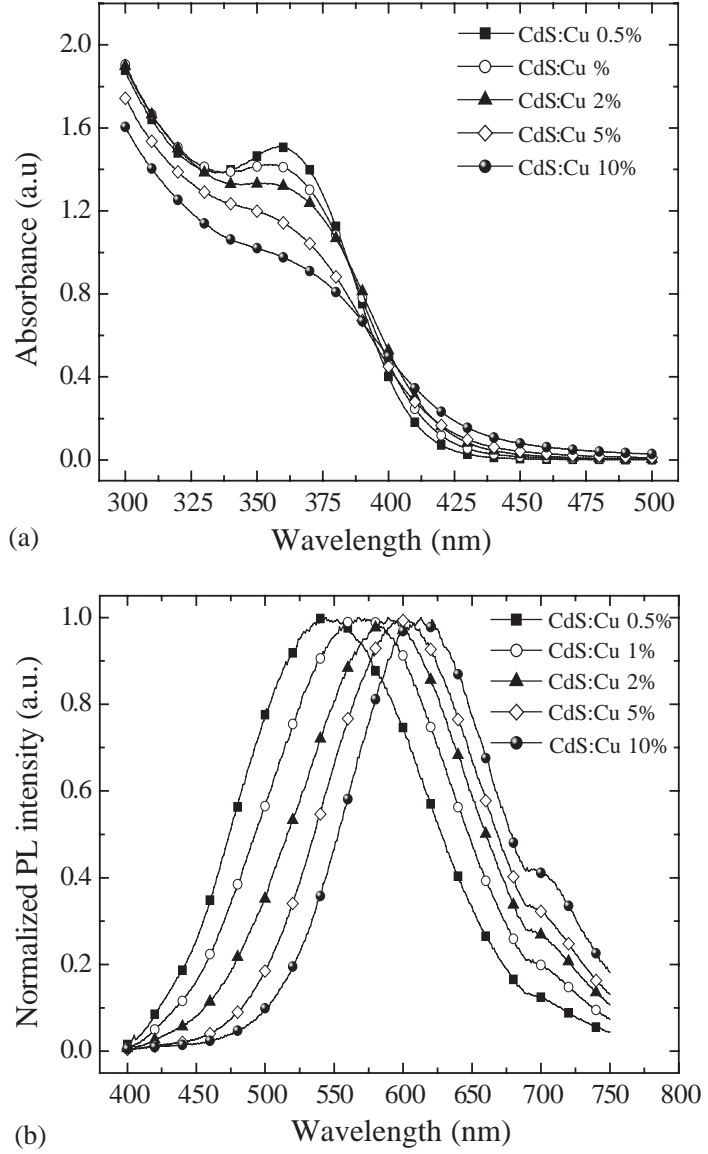

Fig. 10. (a) Absorption and (b) emission spectra ( $\lambda_{\mathrm{ex}}=$ $350 \mathrm{~nm})$ of $\mathrm{CdS}: \mathrm{Cu}(0.5-10 \% \mathrm{Cu}$ added to the solution before particle formation) synthesized in methanol with a MPS concentration of $0.1 \mathrm{M}$.

bandgap but when $\mathrm{Cu}$ is incorporated in the $\mathrm{CdS}$ nanocrystals it serves as a recombination center situated in the bandgap and a red-shifted emission is observed. In Fig. 9 the absorption and emission spectra are depicted for samples with synthesized with various $\mathrm{Cu}$ concentration. The concentrations indicated are concentrations present in the reaction mixture, in $\mathrm{mol} \%$ relative to $\mathrm{Cd}$. The actual concentrations of $\mathrm{Cu}$ incorporated in the CdS nanocrystals will be significantly lower [23]. The absorption and emission spectra show that incorporation of $\mathrm{Cu}$ does not shift the absorption onset, as expected, but does cause a red shift of the emission. For the lower $\mathrm{Cu}$ concentrations the emission spectrum is broadened since 
donor-acceptor emission also from undoped CdS quantum dots is observed. For the sample with $10 \% \mathrm{Cu}$ in the reaction mixture, the emission consists almost exclusively of the red $\mathrm{Cu}$-related emission. The life time of this emission is $2 \mu \mathrm{s}$ at $4 \mathrm{~K}$ and decreases to about $200 \mathrm{~ns}$ at room temperature. A similar behavior for the life time of the $\mathrm{Cu}$-related emission has previously been observed for $\mathrm{ZnSe:Cu}$ [18]. The quantum efficiency of the Cu-related emission is low at room temperature (about 1\%). This makes the red shifted emission less useful for application where a high quantum yield is required.

\section{Conclusions}

A simple synthesis method has been reported for efficiently luminescing CdS quantum dots using MPS as a capping agent. The particle size can be controlled over a large size range $(2-6 \mathrm{~nm})$ by varying the MPS concentration. The size and temperature dependence of the luminescence and the luminescence life time are reported and explained by a model based on a donor-acceptor type luminescence. Tuning of the emission color is possible via particle size (MPS concentration) or by doping with $\mathrm{Zn}$ or $\mathrm{Cu}$. As a result efficiently luminescing quantum dots may be obtained with different emission wavelengths. The use of MPS as capping provides the quantum dots with a precursor for growth of silica at the surface which is useful to incorporate the quantum dots in a silica shell or in a silica matrix.

\section{Acknowledgements}

This work was financially supported by Utrecht University within the 'breedte-strategie' program 'Physics of Colloidal Matter'.

\section{References}

[1] A.P. Alivisatos, J. Chem. Phys. 100 (1996) 13226.

[2] L.E. Brus, J. Chem. Phys. 90 (1986) 2555.

[3] M. Bruchez Jr., M. Moronne, P. Gin, S. Weiss, A.P. Alivisatos, Science 281 (1998) 2013.

[4] W.C.W. Chan, S. Nie, Science 281 (1998) 2016.

[5] C.B. Murray, D.B. Norris, M.G. Bawendi, J. Am. Chem. Soc. 115 (1993) 8706.

[6] D.V. Talapin, S. Haubold, A.L. Rogach, A. Kornowski, M. Haase, H. Weller, J. Phys. Chem. B 105 (2001) 2260.

[7] G.P. Mitchell, C.A. Mirkin, R.L. Letsinger, J. Am. Chem. Soc. 121 (1999) 8122.

[8] H. Mattoussi, J.M. Mauro, E.R. Goldman, G.P. Anderson, V.C. Sundar, F.V. Mikulec, M.G. Bawendi, J. Am. Chem. Soc. 122 (2000) 12142.

[9] B. Dubertret, P. Skourides, D.J. Norris, V. Noireaux, A.H. Brivanlou, A. Libchaber, Science 298 (2002) 1759.

[10] W.J. Parak, R. Boudreau, M. Le Gros, D. Gerion, D. Zanchet, C.M. Micheel, S.C. Williams, A.P. Alivisatos, C. Larabell, Adv. Mater. 14 (2002) 882.

[11] M.A. Correa-Duarte, M. Giersig, L.M. Liz-Marzan, Chem. Phys. Lett. 286 (1998) 497.

[12] T. Vossmeyer, L. Katsikas, M. Giersig, I.G. Popovic, K. Diesner, A. Chemseddine, A. Eychmüller, H. Weller, J. Phys. Chem. 98 (1994) 7665.

[13] A. van Dijken, A.H. Janssen, M.H.P. Smitsmans, D. Vanmaekelbergh, A. Meijerink, Chem. Mater. 10 (1998) 3513.

[14] S. Drouard, S.G. Hickey, D.J. Riley, Chem. Comm. 1 (1999) 67.

[15] A. Hässelbarth, A. Eychmüller, H. Weller, Chem. Phys. Lett. 202 (1993) 271.

[16] A. Eychmüller, A. Hässelbarth, L. Katsikas, H. Weller, J. Lumin. 48-49 (1991) 745.

[17] Y.P. Varshni, Physica 34 (1967) 149.

[18] J.F. Suyver, T. van der Beek, S.F. Wuister, J.J. Kelly, A. Meijerink, Appl. Phys. Lett. 79 (2001) 4222.

[19] N. Chestnoy, T.D. Harris, R. Hull, L.E. Brus, J. Phys. Chem. 90 (1986) 3393.

[20] C. De Mello Donega, A. Meijerink, G. Blasse, J. Phys. Chem. Solids 56 (1995) 673.

[21] D.V. Lang, C.H. Henry, Phys. Rev. Lett. 35 (1975) 1525.

[22] H. Yukselici, P.D. Persans, T.M. Hayes, Phys. Rev. B 52 (1995) 11763.

[23] A.A. Bol, J.A. Bergwerff, J. Ferwerda, A. Meijerink, J. Lumin. 99 (2002) 325. 